\title{
ORIGINAL ARTICLE \\ Genetic and environmental determinants of unreduced gamete production in Brassica napus, Sinapis arvensis and their hybrids
}

\author{
D Sora ${ }^{1}, \mathrm{P}$ Kron $^{2}$ and BC Husband ${ }^{3}$ \\ Unreduced gametes, sperm or egg cells with the somatic chromosome number, are an important mechanism of polyploid \\ formation and gene flow between heteroploid plants. The meiotic processes leading to unreduced gamete formation are well \\ documented, but the relative influence of environmental and genetic factors on the frequency of unreduced gametes remain \\ largely untested. Furthermore, direct estimates of unreduced gametes based on DNA content are technically challenging and, \\ hence, uncommon. Here, we use flow cytometry to measure the contribution of genetic (hybridization) and environmental \\ (nutrient limitation, wounding) changes to unreduced male gamete production in Brassica napus, Sinapis arvensis and two \\ hybrid lines. Treatments were applied to greenhouse grown plants in a random factorial design, with pollen sampled at two time \\ intervals. Overall, the frequency of unreduced gametes averaged $0.59 \%$ (range $0.06-2.17 \%$ ), plus a single outlier with $27 \%$. \\ Backcrossed hybrids had 39 to $75 \%$ higher unreduced gamete production than parental genotypes, averaged across all \\ treatments, although the statistical significance of these differences depended on sampling period and wounding treatment. \\ Unreduced gamete frequencies were higher for the second sampling period than the first. There were no direct effects of \\ wounding or nutrient regime. Our results indicate that both genetic and environmental factors can induce increased unreduced \\ gametes, highlighting the potential importance of environmental heterogeneity and genetic composition of populations in driving \\ polyploid evolution.
}

Heredity (2016) 117, 440-448; doi:10.1038/hdy.2016.69; published online 31 August 2016

\section{INTRODUCTION}

Polyploidy, the possession of more than two complete sets of chromosomes, is associated with early diversification of eukaryotes (Katz, 2012) and is an important means of ecological divergence and speciation in plants (Rieseberg and Willis, 2007). One of the most prevalent modes of polyploid plant formation is through the union of unreduced gametes (Harlan and deWet, 1975; Ramsey and Schemske, 1998), the sperm and egg cells whose nuclei contain the same chromosome number as somatic nuclei. In addition to this role in neopolyploid formation, unreduced gametes also facilitate gene flow across different cytotypes and species. This is because unreduced gamete chromosome numbers may match those of reduced gametes in higher cytotypes, resulting in progeny that have even chromosome copy numbers and that are therefore more likely to be reproductively viable (Kron and Husband, 2009; Paun et al., 2009). Evidence of gene flow between cytotypes has been demonstrated in natural populations of Dactylis glomerata (Lumaret and Barrientos, 1990) as well as between species of Taraxacum spp. (Menken et al., 1995), likely as a product of unreduced gametes. Unreduced gametes have also been useful in plant breeding to introgress desirable genetic traits between different cytotypes (Dewitte et al., 2012).

Unreduced gametes have been documented in over 34 plant families (Bretagnolle and Thompson, 1995; Dewitte et al., 2012) and at rates ranging from 0.0 to $81.1 \%$ of all gametes produced (Ramsey and Schemske, 1998). They are formed through meiotic abnormalities, such as pre-meiotic doubling, premature cytokinesis, mutation in synapsis, lack of spindle fiber segregation and post-meiotic fusion (Bretagnolle and Thompson, 1995; De Storme and Geelen, 2013), and through defects in the mitotic cell cycle involving poor control of cyclin-dependent kinases (Brownfield and Köhler, 2011). However, although our knowledge of these formation mechanisms has grown, our understanding of the role of unreduced gametes in polyploid evolution still requires a greater knowledge of the incidence, magnitude and determinants of unreduced gamete production. There is still little direct evidence identifying key conditions where unreduced gamete formation is favored, and the underlying determinants of the rate of unreduced gamete production at individual and population levels, and the interactions between these determinants, have rarely been investigated empirically (Ramsey and Schemske, 1998; Soltis et al., 2010; Mason et al., 2011; Mason and Pires, 2015).

A variety of genetic and environmental factors have been linked to high rates of unreduced gamete production, but our understanding of the relative importance of genotype and environment remains incomplete. One major genetic factor implicated is hybridization (Ramsey and Schemske, 1998) that is thought to increase unreduced

${ }^{1}$ Department of Biology, Bioscience Complex, Queen's University, Kingston, Ontario, Canada; ²Department of Integrative Biology, University of Guelph, Guelph, Ontario, Canada and ${ }^{3}$ Department of Integrative Biology, University of Guelph, Guelph, Ontario, Canada

Correspondence: D Sora, Department of Biology, Bioscience Complex, Queen's University, Kingston, Ontario K7L 3N6, Canada.

E-mail: dylan.sora@gmail.com

Received 1 March 2016; revised 8 July 2016; accepted 13 July 2016; published online 31 August 2016 
gamete production as newly formed plants often have noncomplementary chromosomes, resulting in irregular pairing and chromosome segregation failure during meiosis (Nelson et al., 2009; Ainouche and Jenczewski, 2010; De Storme and Geelen, 2013). In addition, genes that code for proteins regulating meiosis, and therefore unreduced gamete production, have been identified in plants, and these genes may vary among individuals and populations (Brownfield and Köhler, 2011). Finally, plants with an odd number of paired chromosomes, such as triploids, may have irregular pairing in meiosis resulting in increased unreduced gamete formation compared with plants with an even number of chromosomes, such as diploids (Dweikat and Lyrene, 1988).

Adverse environmental conditions may also affect unreduced gamete production by interfering with cellular processes associated with meiosis (Ramsey and Schemske, 1998). The most frequently cited method of inducing unreduced gametes through alteration of environmental conditions is the cycling of hot and cold temperatures (Mason et al., 2011; De Storme et al., 2012). This approach has been used extensively by potato breeders to produce and introgress desirable genes from diploid into tetraploid lineages (Dewitte et al., 2012). Although less intensively studied, nutrient deficiency has also been shown to delay or interfere with formation of integral proteins involved with meiosis initiation (Kimble, 2011) and has been implicated in increased unreduced gamete production (Grant, 1952). Herbivory has also been associated with elevated rates of unreduced gametes when direct floral damage occurs (Kostoff and Kendall, 1929, 1930; Kostoff, 1933) but the effects of herbivory on unreduced gamete production have received little or no attention since these early studies. The studies linking increased unreduced gamete formation to nutrient limitation and herbivory have thus far been largely anecdotal, without using accurate techniques to measure rates of unreduced gametes (Kostoff, 1933; Grant, 1952).

Experimental studies to determine the causes of unreduced gamete production have been limited in part by the technical challenges involved in detecting these typically rare events (Ramsey and Schemske, 1998). Traditionally, pollen size and microspore analysis have been used to measure the frequency of pollen with unreduced gametes (hereafter referred to as 'unreduced pollen'). Estimates based on pollen size rely on finding oversized pollen grains, typically $30-40 \%$ larger than reduced pollen (Ramsey, 2007). This technique is limited by the number of pollen grains that can be visualized and measured when done microscopically, although this limitation is overcome when volumetric measurement is used (De Storme et al., 2013). However, size-based methods may be inaccurate in species with substantial pollen size variation unrelated to ploidy, or ones in which there is little size differentiation between 1 and $2 \mathrm{~N}$ pollen (Bretagnolle and Thompson, 1995). Alternatively, microspores (sporads) from postmeiotic pollen mother cells (PMC) are visualized to determine the frequency of dyads, mother cells containing two rather than four sister cells with twice the nuclear DNA content of typical tetrads (Mason et al., 2011). The presence of dyads indicates that pollen mother cells failed to complete meiosis, and is a precursor to unreduced gametes (Dewitte et al., 2009). This method is limited by how many cells can be observed, difficulties in distinguishing dyads from cells still undergoing meiosis (Dewitte et al., 2012) and tetrads whose four cells appear as two because of their orientation in two-dimensional microscopy (Bremer, 1963). Although not without its own limitations, flow cytometry (FCM), which directly measures the DNA content of pollen nuclei, overcomes some of the shortcomings of other methods in species meeting criteria for its use (Kron and Husband, 2012, 2015).
This technique allows for the analysis of tens of thousands of pollen nuclei per plant, giving finer resolution in detecting the typically rare nonreduction events (Kron and Husband, 2012). Because estimates are based on nuclei DNA contents, this technique measures unreduced gamete production among viable pollen grains only, and therefore provides a 'realized' estimate of unreduced male gamete production.

In this study we examine the contribution of genetic and environmental factors to unreduced pollen production in Brassica napus (canola), Sinapis arvensis (wild mustard; syn. B. kaber) and hybrid lines backcrossed to $B$. napus. B. napus is a naturally occurring allotetraploid plant, widely grown in the northern hemisphere for cooking oil production; S. arvensis is a diploid, self-incompatible, weedy plant found in recently disturbed fields across the northern hemisphere (Warwick et al., 2003). We used these species to understand the determinants of unreduced gamete production for three reasons. First, there is concern for the negative consequences of hybridization and gene flow from transgenic Brassica crops to weedy Brassicaceae relatives through unreduced gametes (Warwick et al., 2003). Although several studies (Ramsey and Schemske, 1998) have looked at the effects of F1 hybridization on unreduced gametes, ongoing unreduced gamete production in subsequent hybrid generations may also contribute to gene flow. Using backcrossed lineages allows us to assess how recurrent backcrossing affects rates of unreduced gametes, as repeated backcrossing may reduce the amount of genetic and chromosomal mismatches and therefore associated meiotic problems. Second, B. napus and other Brassica species have been previously used in studies that identify rates of unreduced gametes using pollen mother cells analysis (Mason et al., 2011), providing a frame of reference for our results. Third, species in the Brassicaceae have pollen nuclei morphology conducive to the correction for aggregates when analyzing flow cytometric data, a crucial step when quantifying unreduced gametes cytometrically (Kron and Husband, 2015).

Here, we assess the effects of hybridization across multiple generations of backcrossing, nutrient limitation and leaf wounding (simulated herbivory) on unreduced gamete production in these species. We applied these treatments factorially allowing us to assess the impacts of environment and genetics both independently and in combination to determine their relative importance on unreduced gamete production. Using FCM to estimate frequencies of unreduced gametes, we address the following specific questions: (1) what are the overall rates of unreduced gametes in these species; (2) is there evidence for a genetic effect (hybridization), environment stress effects or both; and (3) are environmental factors additive or is there an interaction between genetic and environmental factors? We hypothesized that adverse genetic and environmental circumstances will act both independently and additively to increase the frequency of gametic nonreduction events in meiosis. We therefore predicted that hybrids and plants subject to nutrient limitation and leaf wounding would produce a higher frequency of unreduced gametes than controls, with hybrids subjected to both environmental treatments producing the highest rates. Because published studies using FCM to estimate unreduced gamete production are still limited in number, we also evaluated two aspects of the methodology. We quantified the effects (and therefore importance) of rarely applied corrections for aggregates and somatic tissue contamination on flow cytometric unreduced gamete estimates, and assessed how rates of unreduced gametes measured using FCM relate to pollen size, a widely used indicator of unreduced gamete production. 


\section{MATERIALS AND METHODS}

\section{Genetic and environmental factors}

We examined the effects of genetic and environmental determinants of unreduced pollen gamete production using a full factorial design where nutrient limitation, wounding and control treatments were performed on two parental and two backcrossed hybrid lineages.

\section{Genetic lines}

To test the effect of hybridization across generations of backcrossing we used four plant lineages: (1) tetraploid B. napus 'Westar', (2) naturally occurring diploid S. arvensis, (3) a fourth-generation backcrossed hybrid (BC4) of B. napus $\times$ S. arvensis and (4) a seventh-generation backcrossed hybrid (BC7). The development of these hybrids is described in Jugulam et al. (2015). B. napus 'Westar' was used in the creation of the BC4 and BC7 backcross hybrids and our $S$. arvensis were grown from seed from the same wild population as progenitors of these lines (C Hall, personal communication). Jugulam et al. (2015) reported that the BC4 plants had 2C DNA contents consistent with a mix of tetraploids and near-tetraploid aneuploids $\left(2.4-2.5 \mathrm{pg} 2 \mathrm{C}^{-1}\right)$, whereas $\mathrm{BC} 7$ plants had tetraploid DNA contents $\left(2.4 \mathrm{pg} 2 \mathrm{C}^{-1}\right)$. We estimated 2C DNA content for a subset of our $\mathrm{B}$. napus, $\mathrm{BC} 4$ and $\mathrm{BC} 7$ plants, using standard onestep flow cytometric protocols for leaf tissue with LB01 buffer (Doležel et al., 1992) and an internal DNA content standard of Solanum lycopersicum 'Stupické polní rané (1.96 pg 2C $\mathrm{C}^{-1}$, Doležel et al., 2007). Our mean DNA contents were in good agreement with those of Jugulam et al. (2015): B. napus $2.47 \pm 0.014 \mathrm{pg} 2 \mathrm{C}^{-1}, N=26 ; \mathrm{BC} 42.49 \pm 0.012 \mathrm{pg} 2 \mathrm{C}^{-1}, N=67$; and $\mathrm{BC} 7$ $2.48 \pm 0.008 \mathrm{pg} 2 \mathrm{C}^{-1}, N=73$; values shown \pm s.e. Although backcrossed hybrids showed the same phenotype as B. napus in terms of DNA content, silique (fruit) production and appearance, the backcrossed lineages displayed the pesticide-resistant phenotype found in the parental $S$. arvensis (Jugulam et al., 2015), indicating the continuing presence of S. arvensis genes in the hybrids.

\section{Environmental factors}

To test the effect of environment on unreduced gamete production, we applied nutrient limitation and leaf wounding treatments. Plants were grown in a glasshouse in the University of Guelph Phytotron $\left(14 \mathrm{~h}\right.$ days at $23-25^{\circ} \mathrm{C}$, nights at $19-21^{\circ} \mathrm{C}$ ). Seeds were individually sown in $1.67-1$ pots with nutrientdeficient Sunshine LA2 soil (SunGrow, Vancouver, BC, Canada) and fully randomized by each treatment combination on a single bench. Once a week until the first flower buds appeared, we watered all plants with reverse osmosis water containing Plant-Prod Solutions $18-9-18 \mathrm{pH}$ reduced fertilizer (Plant Product Co. Ltd, Ancaster, ON, Canada) at a concentration of 200 p.p.m. nitrogen. Once the first flower buds had appeared, we watered half of the plants per line weekly with the same fertilized water ('nutrient supplemented'), whereas the other half received only reverse osmosis water ('nutrient limited'). During the same time period, half of all plants received a wounding treatment, where we pierced the two largest unwounded leaves throughout the leaf surface twice a week, with a frog pin, to simulate leaf damage caused by the various insect herbivores that feed on B. napus (Gulden et al, 2008). We applied nutrient and wounding treatments in a fully random manner in all combinations to both plant parental lines and backcross hybrid lineages. These treatments were applied at rates low enough that the plants would still flower but high enough that plants experienced visible damage in the form of purple leaf discoloration in both treatments. Each line $\times$ nutrient $\times$ wounding treatment combination initially had from 15 to 20 plants for an expected total of 300 plants. B. napus and hybrid lineages were grown in the fall of 2013. S. arvensis failed to germinate in 2013 and instead was tested in the winter of 2014 along with five B. napus control plants. These five control plants were compared with the main B. napus plants to test for differences in unreduced gamete production between fall 2013 and winter 2014.

Both environmental treatments were applied continuously after the onset of initial flower bud formation and were continued until the final pollen collection. We delayed application of these treatments to ensure they would not prevent flowering, but applied them early enough to ensure that the treatments coincided with meiosis early in bud formation (Scott et al., 1991). Specifically, when several plants had begun to initiate buds, nutrient and wounding treatments were started on all plants. After 10 days of treatment, we marked flower bud clusters containing only buds less than $\sim 5 \mathrm{~mm}$ in length. Only flowers developing from these clusters or later ones were used in the study to ensure that meiosis had occurred after some exposure to the environmental treatments. We observed the development time of 23 buds from the 1-2 mm stage (meiotic; Scott et al., 1991) in order to estimate the time between meiosis and anthesis. We collected pollen for all flowering plants concurrently in two distinct time periods: 17 days after the start of the treatments and 31 days after starting treatments. Based on our observations of development time from meiosis to anthesis, we estimate that flowers sampled at 17 days were smaller than $2 \mathrm{~mm}$ (pre-meiotic) for 7 to 10 days after treatments began, and 31 day flowers had been treated 21 to 24 days before meiosis. We collected pollen for flow cytometric analysis from every marked flowering plant by removing all stamens with dehisced anthers from 4 to 6 flowers. Because of variation in flowering start and end times and some limited mortality, not all plants were sampled in one or both sampling periods (Supplementary Table S1). These anthers were stored inside microcentrifuge tubes by dehydrating them with silica gel and refrigerating them $\left(4^{\circ} \mathrm{C}\right)$ for a minimum of $24 \mathrm{~h}$ and a maximum of 2 months.

We used a multifactorial repeated measures analysis of variance (ANOVA), using only those plants for which we were able to obtain an unreduced gamete estimate in both sampling periods ( $u$; see next section). Unreduced gamete proportion was logit-transformed. We tested normality of residuals using the Shapiro-Wilk W-test and homoscedasticity of variances using Bartlett's test. Sampling period, lineage, nutrient treatment and wounding were included as fixed effects. When fixed effects were significant we performed post hoc analyses using Tukey's HSD (honest significant difference). All statistical tests were performed with JMP statistical software (version 8.0, 2009, SAS Institute, Inc., Cary, NC, USA), $\alpha=0.05$.

To provide an additional test of the impact of nutrient limitation and wounding on reproduction, we counted the total number of siliques per plant for B. napus and hybrid plants during the second (31-day) sampling period. B. napus is self-compatible, as are some of its hybrids (Gulden et al., 2008), and siliques are produced by autogamous self-fertilization in the greenhouse. S. arvensis is an obligate outcrossing species and therefore did not produce siliques.

\section{Unreduced gamete estimation and corrections}

We estimated the proportion of pollen grains with unreduced gametes using the basic methods for pollen nuclei extraction, flow cytometric measurement of nuclear DNA content, and initial data analysis (nuclei number counting, debris and aggregate gating) as described in Kron and Husband (2012,2015). Briefly, we vortexed stamens in LB01 buffer, passed the buffer through a $100 \mu \mathrm{m}$ CellTrics filter (Sysmex America, Inc., Mundelein, IL, USA) to remove somatic tissue, captured the pollen on a $20 \mu \mathrm{m}$ filter and burst the pollen by rubbing it gently against the filter with a blunt rubber probe. Released nuclei were washed through the filter using $50 \mathrm{ml}$ of LB01 buffer with $100 \mu \mathrm{m} \mathrm{ml}^{-1}$ propidium iodide and $50 \mu \mathrm{m} \mathrm{ml}^{-1}$ of RNAse. We stained samples with propidium iodide for 20 to $60 \mathrm{~min}$ and analyzed using a BD FACSCalibur flow cytometer (BD Biosciences, San Jose, CA, USA). We analyzed these data using CellQuest Pro software (version 6.0, 2007, BD Biosciences). We gated out debris using a scatterplot of fluorescence height (intensity), measured with the $670 \mathrm{~nm}$ photodetector, plotted against the parameter of interest for DNA content measurement, fluorescence area measured at $485 \mathrm{~nm}$. We counted events making up the $1 \mathrm{C}$ peaks (reduced gamete nuclei) and the $2 \mathrm{C}$ peaks following manual gating of the $1 \mathrm{C}$ and $2 \mathrm{C}$ clusters on a plot of fluorescence area $(485 \mathrm{~nm}$ propidium iodide) versus side scatter. Events in the $2 \mathrm{C}$ cluster were then divided into single nuclei with $2 \mathrm{C}$ DNA content (putative nuclei from pollen with unreduced gametes) and aggregates (doublets of two 1C nuclei) using pulse analysis, a method that discriminates doublets and singlets based on the relative values of fluorescence height and area in the region of interest.

Following the gating of doublets from the $2 \mathrm{C}$ peak, we did an additional correction for possible somatic tissue contamination. This correction was motivated by the observation that some samples had $4 \mathrm{C}$ events at too high a frequency to be explained as $2 \mathrm{C}+2 \mathrm{C}$ doublets, although still at low numbers (10 or fewer 4C events in $68 \%$ of all samples, in samples with 1C nuclei 
numbers typically in the $20000-30000$ range; $4 \mathrm{C}$ events exceeded 30 in only $7 \%$ of samples). Because endopolyploidy (the occurrence of more than one nuclear ploidy within tissues) is common in Brassicaceae (Barow and Jovtchev, 2007), we hypothesized that these could be $4 \mathrm{C}$ somatic nuclei from pieces of the stamens passing through the $100 \mu \mathrm{m}$ prefilter. In this case, $2 \mathrm{C}$ somatic nuclei could also be present, inflating the unreduced gamete estimate. We collected four samples of 36 stamens from each line (B. napus, S. arvensis, BC4 and BC7; the four samples were from different plants for all except $S$. arvensis, for which two of the four were from the same plant). We vortexed the stamens in LB01 to remove most of the pollen, placed the stamens on $20 \mu \mathrm{m}$ filters and processed them as if they were pollen samples (that is, rubbed them on the filter, rinsed the sample with $0.5 \mathrm{ml} \mathrm{LB01}$ buffer with propidium iodide and RNAse, tested and analyzed them using the methods described above). We calculated the ratio of $2 \mathrm{C}$ nuclei numbers to $4 \mathrm{C}$ nuclei numbers, found the mean ratio for each line and designated this mean ratio as the somatic correction factor for that line (SCF). For each sample, we estimated the number of contaminating $2 \mathrm{C}$ somatic nuclei as $\mathrm{SCF} \times$ (number of $4 \mathrm{C}$ events), and subtracted this from the number of $2 \mathrm{C}$ nuclei. Note that this method relies on endopolyploidy in the source tissue of the contamination, as the $4 \mathrm{C}$ events are used as indicators of, and predictors for the numbers of, contaminating $2 \mathrm{C}$ nuclei. Following somatic correction we calculated the proportion of unreduced gamete nuclei as $u=(2 \mathrm{C}$ nuclei $) /(1 \mathrm{C}$ nuclei $+2 \mathrm{C}$ nuclei).

To assess the impact of our corrections for aggregates and somatic tissue, we repeated the main ANOVA using uncorrected data and data corrected for aggregates only. We compared these analyses to determine whether the corrections had a qualitative impact on our results, that is, whether the corrections changed any assignments of statistical significance (relative to uncorrected data) and thereby influenced our general conclusions. We also calculated mean $u$ for all lines pooled with no corrections, doublet correction only and doublet plus somatic correction to assess the quantitative impact of the corrections on absolute unreduced gamete estimates.

\section{Pollen size comparison to FCM}

We compared mean pollen size with the proportion of unreduced gametes estimated by FCM to test the hypothesis that cytometric estimates of unreduced gamete proportions should be positively correlated with mean pollen size (because the presence of larger, unreduced pollen would inflate the mean). For B. napus, $\mathrm{BC} 4$ and $\mathrm{BC}$, while collecting pollen for $\mathrm{FCM}$, we collected two additional anthers from each plant for pollen size analysis and refrigerated them in $70 \%$ ethanol for 3 months. We tested a subset of these consisting of 72 plants with the highest and lowest estimates of unreduced gametes, as well as one randomly selected plant, from each lineage $\times$ environmental treatment time treatment for pollen diameter testing. Pollen diameter was tested from the two extremes of unreduced gamete production to ensure that the full range of estimates was present in the correlation analysis. Because the objective of this test was not to independently estimate absolute unreduced gamete production using pollen size but rather to test for a correlation between mean pollen size and flow cytometric estimates of unreduced gametes, we did not consider it necessary to test all lineages. We therefore only collected pollen for this test during the B. napus hybrid stage of the study.

We measured pollen diameter using a Multisizer 3 Coulter Counter (Beckman Instruments, Fullerton, CA, USA). We placed tubes with pollen stored in ethanol open inside a fume hood for $24 \mathrm{~h}$ to evaporate the ethanol and then rehydrated with $1 \mathrm{ml}$ of Coulter Counter-specific diluent immediately before testing. To identify the range of pollen diameters we measured 25 pollen grains per plant from 24 plants (8 of each lineage) using a Leica DMR microscope (Leica Microsystems, Wetzlar, Germany) and Openlab software version 4.0.3 (Improvision Inc., Waltham, MA, USA). We ignored smaller collapsed (aborted) pollen. Pollen width ranged from 24 to $40 \mu \mathrm{m}$ and this range was used to establish a measurement range for the Coulter Counter procedure. We vortexed anthers and diluent in $1 \mathrm{ml}$ of diluent and poured into a $30 \mathrm{ml}$ cuvette. We then removed anthers from the cuvette and placed them back into the microcentrifuge tube with $1 \mathrm{ml}$ of diluent. We repeated this process three times per sample. We added $7 \mathrm{ml}$ of diluent for a total volume of $10 \mathrm{ml}$ and tested three $1 \mathrm{ml}$ subsamples from each sample. The mean diameters of all particles in the $24-40 \mu \mathrm{m}$ range were measured, and we averaged the three subsamples to provide a mean pollen diameter for the individual. We used Pearson's correlations to relate flow cytometric unreduced gamete estimates to mean pollen diameter.

\section{RESULTS}

\section{Sample size and unreduced gamete production rates}

In total, we measured unreduced gametes for 177 different plants during one or both sampling periods using FCM. There were 150 plants sampled at 17 days and 110 at 31 days, with 82 plants sampled at both times (after exclusion of 3 samples with nuclei number $<10000)$. Most failures to sample plants at both times were because they had not started flowering at 17 days or had finished by 31 days; our final set of 82 therefore did not include those plants that started flowering the latest or finished flowering earliest. The 82 plants sampled at both time intervals were used for the repeated measures ANOVA, and all subsequent results refer to this set of $82 \times 2$ samples unless otherwise stated. For this data set, there was only one $S$. arvensis plant sampled in each of the nutrient supplemented $\times$ wounded and nutrient supplemented $\times$ nonwounded treatment combinations, but all other lineage $\times$ environmental treatment combinations included from 3 to 10 replicates (Supplementary Table S1).

The percentage of unreduced gametes ranged from 0.065 to $1.95 \%$ (all treatments pooled, data corrected for aggregates and somatic tissue, untransformed data), with a mean of $0.59 \%$ ( $95 \%$ confidence interval $0.55-0.64 \% ; N=164$; mean and confidence interval backtransformed from logit-transformed distribution) (Supplementary Figure S1). When the rest of the samples were considered (that is, those sampled at 17 or 31 days but not at both), two higher percentages of unreduced gametes were also observed: one with $2.17 \%$, and one S. arvensis sample with $26.70 \%$ unreduced gametes (nutrient limited, nonwounded, 31-day treatment). Note that because the plant producing the $26.70 \%$ outlier was not sampled at both time periods, it was not included in the repeated measures ANOVA.

\section{Effects of environmental and genetic treatments}

Our logit-transformed data met assumptions for ANOVA (that is, normality and homoscedasticity of residuals). There were significant effects of sampling period $(P=0.0001)$ and lineage $(P<0.0001)$, as well as significant interactions between sampling period and line $(P=0.0169)$ and wounding treatment and line $(P=0.0419$; Table 1$)$. Generally, the two hybrid lineages (BC4 and $\mathrm{BC} 7)$ produced more unreduced gametes than the parental lines (B. napus and S. arvensis), but the statistical significance of these differences varied in the context

Table 1 Results of repeated measures ANOVA with unreduced gamete proportion (logit-transformed) as the response variable

\begin{tabular}{lcccr}
\hline Factor & d.f. & d.f. denom. & F-ratio & Prob $>F$ \\
\hline Sampling period & 1 & 76 & 16.5595 & 0.0001 \\
Line & 3 & 69 & 11.8821 & $<0.0001$ \\
Nutrients & 1 & 69 & 0.0699 & 0.7923 \\
Wounding & 1 & 69 & 0.1767 & 0.6755 \\
Sampling period $\times$ line & 3 & 76 & 3.6153 & 0.0169 \\
Sampling period $\times$ nutrients & 1 & 76 & 0.0661 & 0.7977 \\
Sampling period $\times$ wounding & 1 & 76 & 0.397 & 0.5305 \\
Line $\times$ nutrients & 3 & 69 & 0.7326 & 0.5361 \\
Line $\times$ wounding & 3 & 69 & 2.883 & 0.0419 \\
Nutrients $\times$ wounding & 1 & 69 & 2.2166 & 0.1411 \\
\hline
\end{tabular}

Abbreviations: ANOVA, analysis of variance; denom, denominator; Prob, probability. 

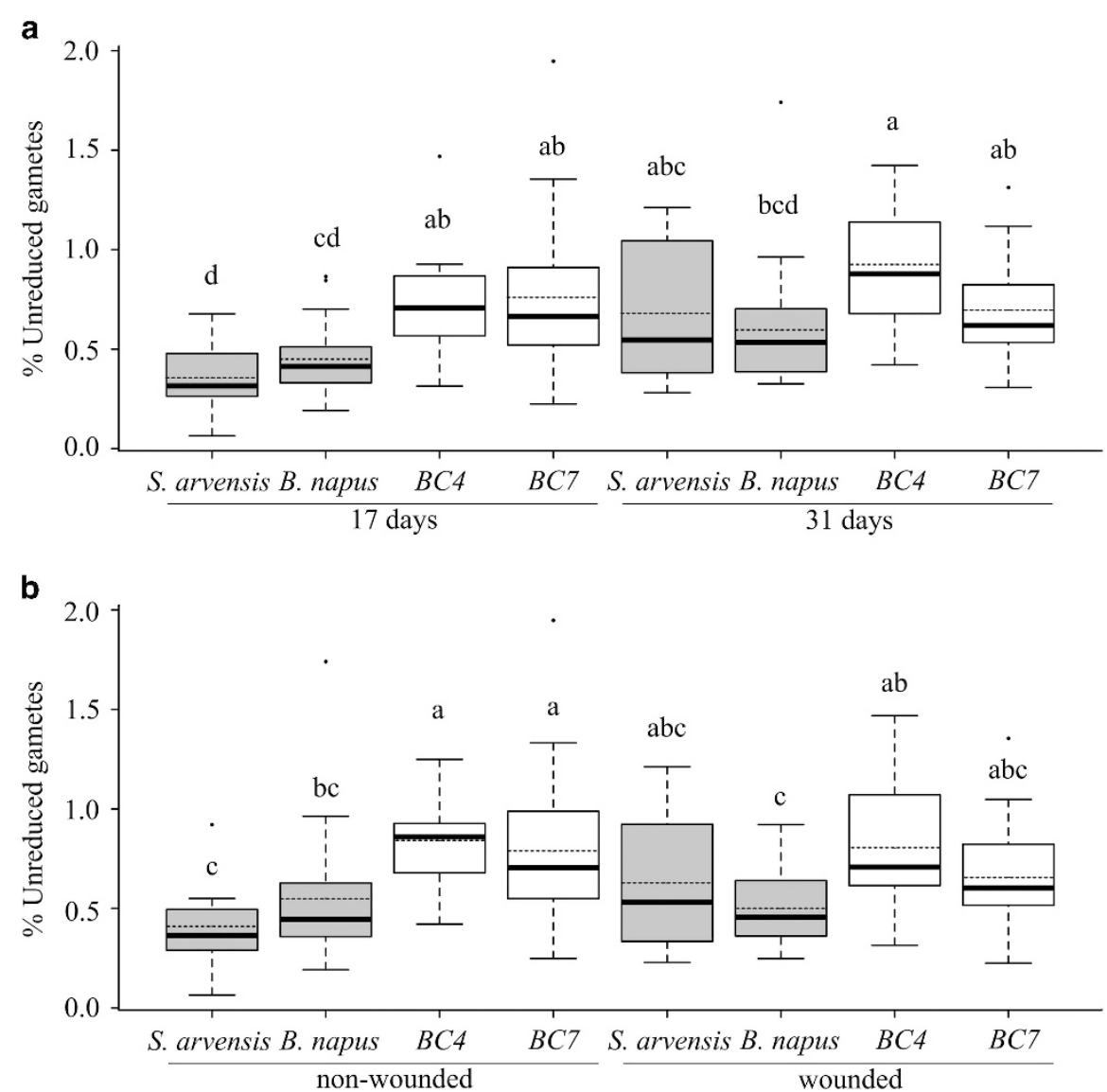

Figure 1 Tukey's boxplots showing untransformed unreduced gamete proportion medians (dark lines) and means (dashed lines) for treatment $\times$ line combinations. (a) Combinations of line and sampling period; (b) line $\times$ wounding treatment. Treatment combinations sharing a letter code within a plot had least square means not significantly different $(\alpha=0.05$; Tukey's HSD test) for the repeated measures ANOVA using logit-transformed data.

of the other treatments. BC4 and BC7 (0.68\%) had significantly more unreduced gametes than both parental lines at 17 days (B. napus $0.42 \%, S$. arvensis $0.32 \%)$. At 31 days, both hybrids still averaged higher than the parental lines (BC4 $0.90 \%$, BC7 0.66\%, S. arvensis $0.63 \%, B$. napus $0.55 \%$ ) but the only statistically significant difference was between BC4 and B. napus (Figure 1a; all values reported here are back-transformed least square means from the ANOVA using logittransformed data; Figure 1 shows means for untransformed data). All lines showed an increase in unreduced gametes over time except BC7 that showed a slight decrease (from 0.68 to $0.66 \%$ ) and, overall, the 31 -day values averaged $34 \%$ higher than the 17 -day values $(0.67 \%$ vs $0.50 \%)$. However, when considered separately by lineage, only the near doubling in S. arvensis $(0.32$ to $0.63 \%)$ was significant. With respect to wounding, $\mathrm{BC} 4$ and $\mathrm{BC} 7$ had significantly more unreduced gametes than both parental lines for nonwounded plants only (Figure 1b). In wounded plants, both hybrids averaged higher than the parental lines but only BC4 was significantly greater than B. napus.

Unreduced gamete production in plants screened at 17 days was correlated with measures for the same plants at 31 days $(P=0.0287$, $r=0.2417$, Pearson's correlation). Significant increases in silique production were observed in nutrient-supplemented plants compared with nutrient-limited plants (ANOVA, $F=14.73$; $P<0.001$ ) but no effect of wounding on fruit production was observed (ANOVA, $F=0.083, P=0.77$; Supplementary Table S2). The control B. napus individuals grown along with the $S$. arvensis in the winter of 2014 showed no difference in mean unreduced gamete production when compared with control plants grown in fall $2013(t=0.15, P=0.84)$.

\section{Effect of corrections on unreduced gamete estimates}

Qualitatively, the corrections of the flow cytometric data for aggregates and somatic tissue had little effect on the results. For uncorrected data, the outcome was the same as for fully corrected data: significant effects were observed for treatment time, line, time $\times$ line and wounding $\times$ line interactions, with the same relationships between factors. Quantitatively, the combined effect of the corrections for aggregates $(1 \mathrm{C}+1 \mathrm{C}$ doublets) and somatic contamination was a reduction in the unreduced gamete estimate from a mean of $0.84 \%$ uncorrected to $0.59 \%$ with both corrections. This reduction was primarily the result of doublet correction that reduced the estimate from 0.84 to $0.63 \%$. Estimates of unreduced gamete proportion before somatic tissue correction were correlated with the proportion of $4 \mathrm{C}$ events in the sample $(P=0.0056, r=0.217)$ but not significantly correlated after somatic tissue correction $(P=0.8401, r=0.0160 ; N=162)$, consistent with the hypothesis that $4 \mathrm{C}$ events indicate the presence of somatic $2 \mathrm{C}$ events. For this correlation test we excluded one of the 82 plants with an unusually low measure of unreduced gametes at 17 days, because inclusion of this plant resulted in nonnormal residuals.

Relationship between pollen size and unreduced gamete estimates Unreduced gamete estimates determined by FCM were positively correlated with mean pollen diameter (Pearson's correlation, $r=0.31$, 


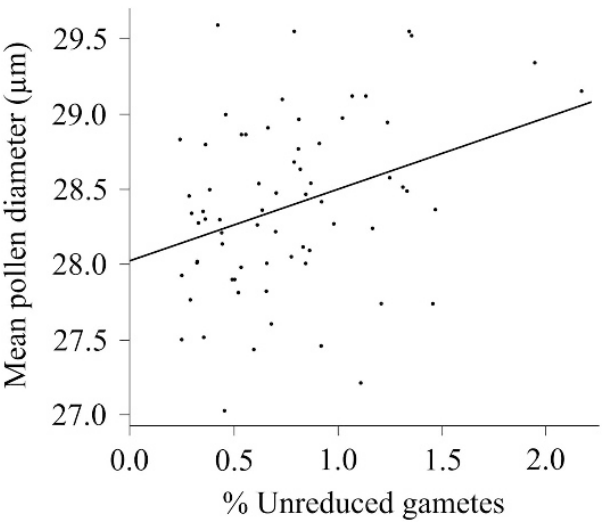

Figure 2 Linear regression showing relationship between mean pollen diameter measured volumetrically and proportion of unreduced gametes measured cytometrically. Mean diameter $=28+47.5 u$ where $u$ is unreduced gamete proportion; $r=0.31, P=0.0055$.

$P=0.0055$; Figure 2). A linear regression with $u$ as the independent variable produces a best-fit line mean diameter $=28.0+47.5 u$. To test whether differences in reduced pollen size between lines may have been confounded with unreduced pollen frequency differences, we performed an analysis of covariance comparing pollen mean diameter between lines with unreduced gamete proportion as a covariate. There was a significant effect of unreduced gamete proportion on pollen mean diameter $(P=0.0025)$ but no differences between lines with respect to pollen size $(P=0.2342)$.

\section{DISCUSSION}

\section{Rates of unreduced gamete production}

We observed a wide spectrum of unreduced gamete frequencies, ranging from 0.06 to $2.17 \%$ with an outlier at $26.70 \%$. The 0.06 to $2.17 \%$ range is consistent with the observation of Ramsey (2007) that 0.1 to $2 \%$ of gametes are expected to be unreduced in nonhybrid populations. As well, the skewed distribution of unreduced gametes, with most individuals having low rates and a few with much higher rates, has been observed before when unreduced gametes have been quantified across large numbers of individuals (Ramsey, 2007; Dewitte et al., 2012). The combination of low-level baseline unreduced gamete production along with a single high-level producer highlights the variable nature of unreduced gamete production that may be a product of environment, development and genetics (Ramsey and Schemske, 1998). Intensive sampling at other scales, including within individuals, could reveal additional patterns, including the possibility that variation in unreduced gamete production may be greatest at the scale of the flower within an individual, as is the case for seed size (Michaels et al., 1988).

\section{Genetic and genomic effects}

Unreduced gamete estimates for individual plants were correlated between the two sampling periods. This result is consistent with a genetic component for the phenomenon, although other factors might explain this pattern (for example, maternal effects, pot-specific environmental variation). The strongest support for genetic or genomic effects, and the most striking result overall, was the higher rate of unreduced gamete production in the two backcross hybrid lineages (BC4 and $\mathrm{BC} 7$ ) compared with the parental lineages (B. napus and $S$. arvensis). This effect was most pronounced at 17 days of treatment time, when both hybrid lineages had higher unreduced gamete estimates than the two parental lineages, but the same pattern was present at 31 days, when $\mathrm{BC} 4$ was still significantly higher than $B$. napus (Figure 1a).

Our results are consistent with several studies documenting increased unreduced gamete production in hybrids (Ramsey and Schemske, 1998). Mason et al. (2011) measured rates of unreduced gametes for three Brassica species and their F1 hybrids using pollen mother cell analysis. They found low mean production in $5 \mathrm{~B}$. napus lines $(0-0.11 \%$, mean $=0.03 \%)$, and higher values in $11 \mathrm{~F} 1$ hybrids between B. napus and two other Brassica species (0.17-3.29\%, mean $=1.55 \%)$. Our estimate for B. napus is also low $(0.48 \%)$, although over four times higher than the highest estimate from Mason et al. (2011). We did not have F1 hybrids, and hence we cannot directly compare F1 values with that of Mason et al. (2011). However, although our four- and seven-generation backcrosses showed lower rates of unreduced gamete production $(\mathrm{BC} 4=0.78 \%, \mathrm{BC} 7=0.67 \%)$ than most of the F1 lines of Mason et al. (2011), our estimates were higher than two of their F1 B. napus $\times$ B. carinata lines $(0.17$ and $0.5 \%)$. These differences could reflect differences in genotype, environment or methods of estimating unreduced gametes.

The higher frequency of unreduced gametes in BC4 relative to BC7 is consistent with the expectation that early-generation hybrids should have increased frequencies of noncomplementary chromosomes and therefore more irregular meiotic behavior than nonhybrids or later-generation backcrosses (Ramsey and Schemske, 1998; Paun et al., 2009). The 2C DNA contents of the BC4 and BC7 lines suggest that the repeated backcrossings with tetraploid $B$. napus has stabilized their chromosome numbers around that of B. napus, despite the fact that the parental lines differ in ploidy. Despite this chromosome number stabilization, and a presumed return to predominant bivalent formation at meiosis, it is possible that structural or genetic incompatibilities persist between the two genomes, and that these may lead to meiotic irregularities. It is also possible that in backcrossed hybrid lineages genotypic interactions not directly attributed to hybridity may be responsible for increased rates of unreduced gametes, perhaps through influences on the meiotic pathway unrelated to chromosomal structural mismatches. For example, S. arvensis plants were grown from variable wild-collected seed. This could potentially result in genotypic interactions unrelated to the hybrid nature of the genome, although our replication should have accounted for such effects. Higher realized frequencies of unreduced gametes in hybrid lines could also arise if higher pollen abortion rates are combined with higher viability of unreduced pollen, as suggested by the relative viabilities of reduced and unreduced cultured microspores from F1 hybrids in one Brassica study (Nelson et al., 2009). Although the precise mechanisms for increased unreduced gamete production in this system remain to be explored, this is to our knowledge the first experimental evidence demonstrating that earlier generation hybrids may have increased unreduced gamete production compared with further backcrossed lineages more similar to one of the parental genomes.

\section{Environmental effects}

There was no overall effect of either nutrient manipulation or wounding on unreduced gamete production, although there was an interaction between wounding and lineage effects. The higher rate in hybrid lines compared with parental lines was strongest and significant only in nonwounded plants, because estimates for hybrids declined with wounding but increased for the nonhybrid S. arvensis (Figure 1b). No significant differences were detected between wounded and nonwounded treatments within any of the four lines. Unreduced gamete estimates were slightly higher for low-nutrient than for 
nutrient-supplemented plants in three of the four lines (all but $S$. arvensis), but again no significant differences were found between nutrient treatments.

The absence of environment effects is at odds with reports for other species. Nutrient limitation has been shown to interfere with meiotic protein initiation and formation in yeast species, Schizosaccharomyces pombe and Saccharomyces cerevisiae (Kimble, 2011). The range of environmental treatments we used was conservative in its application, ensuring that plants would flower, and hence the effect of severe adverse environments may have been underestimated, although purple discoloration of leaves was observed in nutrient-limited plants. There is some evidence from our results that suggest more intense or longer treatments may have resulted in changes in unreduced gamete production. Although increases in unreduced gametes were observed for all environmental treatments from 17 to 31 days (Figure 3), the increases were greater in wounded and low-nutrient treatments relative to their less stressful counterparts, although these differences were not strong enough to generate statistical significance. Longer treatment time might have affected meiosis, in particular nutrient limitation, as there may have been nutrients remaining from the nutrient supplementation given before bud formation (Jackson, 2000). With longer or more intense stress treatments, and continuation of the observed trend, we may have observed significant environmental treatment effects.

Leaf wounding did not affect rates of unreduced gametes. The impact of leaf wounding on meiosis may be even more indirect than that of nutrient level. Interestingly, wounding was the only treatment that did not affect silique production, suggesting that our leaf wounding treatment may not have been strong enough, nor applied at the appropriate time or location, to affect meiosis or reproduction in general. It is noteworthy, however, that although wounding and herbivory are frequently listed as an environmental stressor influencing unreduced gamete production (see, for example, Ramsey and

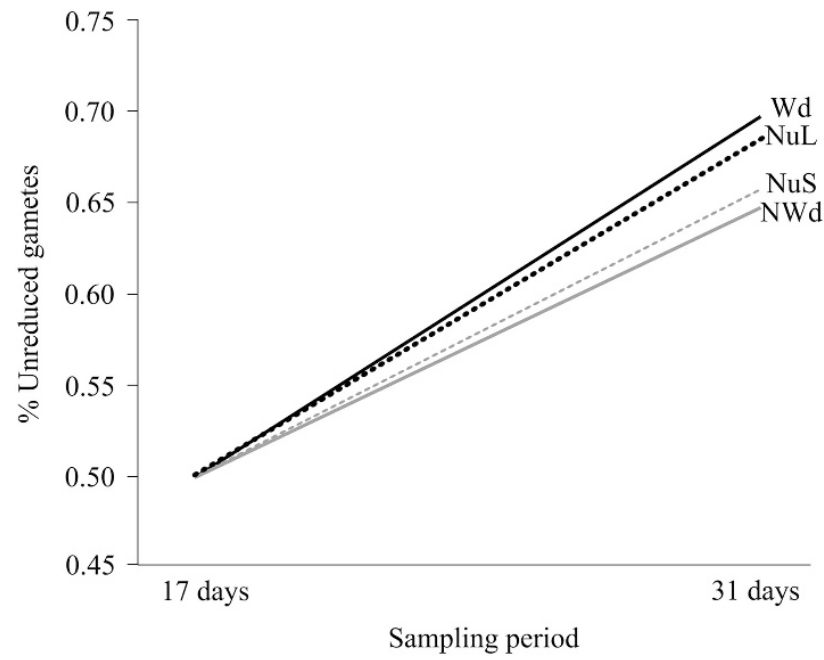

Figure 3 Interaction diagram showing back-transformed least square means (LSMs) for unreduced gamete proportions for each environmental treatment by sampling period combination, averaged across all lineages and the other environmental effect. Dotted lines are nutrient treatments (NuL, nutrient limited; NuS, nutrient supplemented); solid lines are wounding treatments (NWd, nonwounded; Wd, wounded). Treatments considered to be more stressful (Wd, NuL) are black lines, and more benign treatments (NWd, NuS) are gray. At each sampling time (17 days and 31 days), there were no significant differences between Wd and NWd or between NuL and NuS.
Schemske, 1998; De Storme et al., 2012; Mason and Pires, 2015), this observation appears to rest entirely on the early work of Kostoff (Kostoff and Kendall, 1929,1930; Kostoff, 1933). All three of these studies involved plants with serious and direct damage to flower buds by mites (Kostoff and Kendall, 1929,1930) or a virus (Kostoff, 1933). Future studies may detect an increase in unreduced gamete production through direct wounding of flowering buds that are entering meiosis by using mechanical damage, insect herbivores that are known to affect plant reproduction physically or chemically (Hendrix, 1988) or pathogens that influence bud development.

An explanation for the increase in mean unreduced gamete frequency in most treatments from 17 to 31 days is not clear. Plants in all treatments may have become more water and nutrient stressed as they became larger and more pot-bound, or fewer resources may have been devoted to gamete production in the later stages of sexual development. B. napus and hybrid lineages are self-pollinating and self-fertilizing, and during the second sampling period had an average of 54.21 fruits per plant, with few remaining unopened flowers. When embryo and seed production exceeds available resources, remaining resources may be directed toward fruit and seed development to maximize reproductive assurance, for example, by aborting later seeds (Melser and Klinkhamer, 2001). This may draw resources away from gamete production, increasing the number of meiotic errors. In addition, buds developing on primary, secondary and tertiary branches of Arabidopsis thaliana were shown to have different frequencies of crossover events in meiosis (Francis et al., 2007), implicating a higher level of meiotic abnormalities in later developing buds. Finally, it is not impossible that some shift in environmental conditions may have occurred over the 2-week sampling period, despite the consistent environmental controls in the Phytotron. Although developmental stage or nutrient stress may play a role in unreduced gamete production, our data provide no direct evidence of increased unreduced gamete production as a result of nutrient stress or herbivory.

FCM methodology for unreduced gamete estimation

The use of FCM to estimate unreduced gamete production is not always feasible, because high-quality histograms may be difficult to obtain in some species (Kron and Husband, 2012) and because doublet correction may not be practical in species with elongate nuclei (Kron and Husband, 2015). However, using FCM in a system for which these limits either do not apply or can be corrected for, we were able to rapidly determine unreduced male gamete production from a large number of plants, and we were able to estimate frequencies for relatively rare events that may have been missed with methods employing smaller sample sizes. The fact that we had no $0 \%$ estimates is an interesting observation about unreduced gamete production in general, and speaks to the sensitivity of the method in detecting low frequencies of $2 \mathrm{C}$ events. Some caution should be used in interpreting very low (near-zero) values, because the presence of low levels of background noise might inflate such estimates to an extent that can be ignored with higher unreduced gamete estimates (Kron and Husband, 2015). In addition Pan et al., 2004 argue that some $2 \mathrm{~N}$ nuclei in mature pollen could be the product of post-meiotic, mitotic failures. Although our observations from microscopy (proportions of binucleate pollen grains) suggest that such $2 \mathrm{~N}$ nuclei should be rare, these could also contribute significantly to low $2 \mathrm{~N}$ frequencies such as those we observed. Nevertheless, our results demonstrate the power of flow cytometry to detect rare unreduced gamete nuclei after correcting for other kinds of $2 \mathrm{C}$ events. 
As we have demonstrated elsewhere (Kron and Husband, 2015), aggregate correction is important when estimating unreduced gametes with flow cytometry. Although we have stated previously (Kron and Husband, 2012, 2015) that somatic tissue contamination should not be a problem if clean pollen is collected, completely avoiding such contamination may be difficult, especially in species with small flowers where whole flower collection is the most practical approach. In this study, combined correction for doublets and somatic tissue reduced overall unreduced gamete estimates (from 0.84 to $0.59 \%$ ), but did not qualitatively affect the results and conclusions with respect to the effects of our treatments. This result is encouraging if it proves to be the rule in future studies, as these kinds of corrections may not be possible in all cases (for example, doublet correction in species with elongate nuclei, Kron and Husband, 2015; somatic correction in species without somatic endopolyploidy).

Although somatic tissue correction had no impact on our conclusions, there is still reason to believe that it resulted in a small increase in the accuracy of $u$. Before correction, there was a correlation between the proportion of $4 \mathrm{C}$ events in the sample and the unreduced gamete estimate that we hypothesized could occur if $4 \mathrm{C}$ was an indicator of the presence of somatic $2 \mathrm{C}$ nuclei that, in turn, could inflate putative unreduced gamete numbers. Following somatic correction, this correlation was gone, the expected outcome if somatic 2C were excluded, because there is no a priori reason to expect somatic tissue contamination would be correlated with unreduced gamete production. This method therefore appears to be a good approach to correction, but notice that it is only viable in species with endopolyploid somatic tissue, and only when the source tissue of the contamination can be identified, because endopolyploidy may vary between tissues (see, for example, Galbraith et al., 1991). Finally, we cannot completely rule out other explanations for $4 \mathrm{C}$ events correlated with 2C numbers, for example, if $4 \mathrm{C}$ were nuclei from doubleunreduced $(4 \mathrm{~N})$ gametes, and plants producing more of these also produced more unreduced gametes.

Unreduced gamete proportion (estimated using FCM) was correlated with mean pollen size as measured with the Coulter Counter (Figure 2). Although this is the expected relationship (more unreduced gametes means a higher proportion of large pollen grains), the exact relationship we observed is problematic to interpret. The best-fit line (mean diameter $=28.0+47.5 \mathrm{u}$ ) predicts a mean diameter for pollen with reduced gametes that was consistent with our microscopic examinations (mean diameter $=28.0 \mu \mathrm{m}$ when $u=0$ ), but a diameter for pollen with unreduced gametes of $75.5 \mu \mathrm{m}$ (when $u=1$ ). Not only does this not correspond to the expectation that pollen with unreduced gametes should be less than twice the diameter of pollen with reduced gametes (Ramsey, 2007), it also would mean that in samples with unreduced gametes, the larger pollen would not be within the size range we measured (20 to $40 \mu \mathrm{m})$. The observed correlation therefore must be explained in some alternative way. For example, plants with more unreduced gametes may also produce more aneuploid pollen, slightly larger than fully reduced pollen, with nuclear DNA content close enough to $1 \mathrm{C}$ to be interpreted as reduced by FCM.

\section{CONCLUSION}

Our results have implications for our understanding of polyploid evolution. Unreduced gametes are considered an important mechanism of polyploid formation (Harlan and deWet, 1975; Bretagnolle and Thompson, 1995). Our results confirm that unreduced gametes are produced at low but detectable levels in most if not all plants tested, and that individuals with very different, higher levels can occur at low frequencies in a population. The outlier individual that produced $26.70 \%$ was well above the genic rate of mutation reported by Ramsey and Schemske (1998) and may demonstrate the importance of chance events of high unreduced gamete production in polyploid formation. Increased rates of unreduced gametes in hybrid lineages and correlation of unreduced gamete rates from the same individuals across two time periods show support for a genetic influence on unreduced gamete production. The higher hybrid rates are consistent with the idea that populations that experience gene flow from genetically divergent populations or even other species may be more likely to produce polyploids because of increased rates of unreduced gametes caused by their hybrid genomes (Paun et al., 2009) and may indicate the importance of early-generation hybrids in polyploid formation. However, sampling period also affected unreduced gamete production demonstrating environmental components may also influence unreduced gamete formation. We also demonstrate some interaction between environmental and genetic factors, and raise questions about the nature of the claim that herbivory can lead to unreduced gamete production. Taken together, these results indicate that incidence of polyploidy formation may vary widely depending on the genetic or environmental attributes of a population. Our results demonstrate a need for studies investigating the role of environmental variation on unreduced gamete production in natural populations, how phenology is related to unreduced gamete production, the genetics of neopolyploid populations, and the rates of de novo polyploid formation. Moving forward, we have demonstrated that when proper consideration is given to sample quality and corrections for spurious $2 \mathrm{C}$ events, FCM can provide a useful approach to acquiring the large samples sizes and fine-scale measures required for such studies.

\section{DATA ARCHIVING}

Data available from the Dryad Digital Repository: http://dx.doi.org/ $10.5061 /$ dryad. $4 \operatorname{cg} 3 \mathrm{j}$.

\section{CONFLICT OF INTEREST}

The authors declare no conflict of interest.

\section{ACKNOWLEDGEMENTS}

We thank Chris Hall, Asma Ziddulan and Kenny So for providing seeds used and cultivation advice and Mike Mucci and Tannis Slimmon for greenhouse support. We also thank Natural Science and Engineering Research Council of Canada, Canada Research Chair Program and Canadian Foundation for Innovation for financial support.

Ainouche ML, Jenczewski E (2010). Focus on polyploidy. New Phytol 186: 1-4.

Barow M, Jovtchev G (2007). Endopolyploidy in plants and its analysis by flow cytometryln: Flow Cytometry with Plant Cells. In: Dolezel J, Greilhuber J, Suda J (eds), Wiley-VCH Verlag GmbH \& Co. KGaA: Weinheim, Germany, pp 349-372.

Bremer G (1963). Problems in breeding and cytology of sugar cane. VI. Additional contemplations on fertilization and parthenogenesis in Saccharum. Euphytica 12: 178-188.

Bretagnolle F, Thompson JD (1995). Gametes with the somatic chromosome number: mechanisms of their formation and role in the evolution of autopolypoid plants. New Phytol 129: 1-22.

Brownfield L, Köhler C (2011). Unreduced gamete formation in plants: mechanisms and prospects. J Exp Bot 62: 1659-1668.

De Storme N, Copenhaver GP, Geelen D (2012). Production of diploid male gametes in Arabidopsis by cold-induced destabilization of postmeiotic radial microtubule arrays. Plant Physiol 160: 1808-1826.

De Storme N, Geelen D (2013). Sexual polyploidization in plants - cytological mechanisms and molecular regulation. New Phytol 198: 670-684.

De Storme N, Zamariola L, Mau M, Sharbel TF, Geelen D (2013). Volume-based pollen size analysis: an advanced method to assess somatic and gametophytic ploidy in flowering plants. Plant Reprod 26: 65-81. 
Dewitte A, Eeckhaut T, Van Huylenbroeck J, Van Bockstaele E (2009). Occurrence of viable unreduced pollen in a Begonia collection. Euphytica 168: 81-94.

Dewitte A, Van Laere K, Van Huylenbroeck J (2012). Use of $2 \mathrm{n}$ gametes in plant breeding. In: Abdurakhmonov I (ed) Plant Breeding. InTech: Rijeka, Croatia, pp 59-86.

Doležel J, Greilhuber J, Suda J (2007). Estimation of nuclear DNA content in plants using flow cytometry. Nat Protoc 2: 2233-2244.

Doležel J, Sgorbati S, Lucretti S (1992). Comparison of three DNA fluorochromes for flow cytometric estimation of nuclear DNA content in plants. Physiol Plant 85: 625-631.

Dweikat IM, Lyrene PM (1988). Production and viability of unreduced gametes in triploid interspecific blueberry hybrids. Theor App/ Genet 76: 555-559.

Francis KE, Lam SY, Harrison BD, Bey AL, Berchowitz LE, Copenhaver GP (2007). Pollen tetrad-based visual assay for meiotic recombination in Arabidopsis. Proc Natl Acad Sci USA 104: 3913-3918.

Galbraith DW, Harkins KR, Knapp S (1991). Systemic endopolyploidy in Arabidopsis thaliana. Plant Physiol 96: 985-989.

Grant V (1952). Cytogenetics of the hybrid Gilia millefoliataxachilleaefolia. I. Variations in meiosis and polyploidy rate as affected by nutritional and genetic conditions. Chromosoma 5: 372-390.

Gulden RH, Warwick SI, Thomas AG (2008). The Biology of Canadian Weeds. 137. Brassica napus L. and B. rapa L. Can J Plant Sci 88: 951-996.

Harlan JR, deWet JMJ (1975). On Ö. Winge and a prayer: the origins of polyploidy. Bot Rev 41: 361-390.

Hendrix SD (1988). Herbivory and its impact on plant reproduction. In: Lovett Doust J, Lovett Doust L (eds) Plant Reproductive Ecology: Patterns and Strategies. Oxford University Press: New York, pp 246-263.

Jackson GD (2000). Effects of nitrogen and sulfur on canola yield and nutrient uptake. Agron J 92: 644-649.

Jugulam M, Ziauddin A, So KKY, Chen S, Hall JC (2015). Transfer of Dicamba tolerance from Sinapis arvensis to Brassica napus via embryo rescue and recurrent backcross breeding. PLoS One 10: e0141418.

Katz LA (2012). Origin and diversification of eukaryotes. Annu Rev Microbiol 66: $411-427$.

Kimble J (2011). Molecular regulation of the mitosis/meiosis decision in multicellular organisms. Cold Spring Harb Perspect Biol 3: a002683.

Kostoff D (1933). A contribution to the sterility and irregularities in the meiotic processes caused by virus diseases. Genetica 15: 103-114.

Kostoff D, Kendall J (1929). Irregular meiosis in Lycium halimifolium Mill. produced by gall mites (Eriophyes). J Genet 21: 113-115.

Kostoff D, Kendall J (1930). Irregular meiosis in Datura ferox caused by Tetranychus telarius. Genetica 12: 140-144.

Kron P, Husband BC (2009). Hybridization and the reproductive pathways mediating gene flow between native Malus coronaria and domestic apple, M. domestica. Botany 87: 864-874.
Kron P, Husband BC (2012). Using flow cytometry to estimate pollen DNA content: improved methodology and applications. Ann Bot 110: 1067-1078.

Kron P, Husband BC (2015). Distinguishing 2N gamete nuclei from doublets in pollen using flow cytometry and pulse analysis. Cytometry A 87A: 943-957.

Lumaret R, Barrientos E (1990). Phylogenetic relationships and gene flow between sympatric diploid and tetraploid plants of Dactylis glomerata (Gramineae). Plant Syst Evol 169: 81-96.

Mason AS, Nelson MN, Yan G, Cowling WA (2011). Production of viable male unreduced gametes in Brassica interspecific hybrids is genotype specific and stimulated by cold temperatures. BMC Plant Biol 11: 103.

Mason AS, Pires JC (2015). Unreduced gametes: meiotic mishap or evolutionary mechanism? Trends Genet 31: 5-10.

Melser C, Klinkhamer PGL (2001). Selective seed abortion increases offspring survival in Cynoglossum officinale (Boraginaceae). Am J Bot 88: 1033-1040.

Menken S, Smit E, Den Nijs H (1995). Genetical population structure in plants: gene flow between diploid sexual and triploid asexual dandelions (Taraxacum Section Ruderalia). Evolution 49: 1108-1118.

Michaels HJ, Benner B, Hartgerink AP, Lee TD, Rice S, Willson MF et al. (1988). Seed size variation: magnitude, distribution, and ecological correlates. Evol Ecol 2: 157-166.

Nelson MN, Mason AS, Castello M-C, Thomson L, Yan G, Cowling WA (2009). Microspore culture preferentially selects unreduced $(2 n)$ gametes from an interspecific hybrid of Brassica napus L. $\times$ Brassica carinata Braun. Theor Appl Genet 119: 497-505.

Pan G, Zhou Y, Fowke LC, Wang H (2004). An efficient method for flow cytometric analysis of pollen and detection of $2 \mathrm{n}$ nuclei in Brassica napus pollen. Plant cell reports 23: 196-202.

Paun O, Forest F, Fay MF, Chase MW (2009). Hybrid speciation in angiosperms: parental divergence drives ploidy. New Phytol 182: 507-518.

Ramsey J (2007). Unreduced gametes and neopolyploids in natural populations of Achillea borealis (Asteraceae). Heredity 98: 143-150.

Ramsey J, Schemske DW (1998). Pathways, mechanisms, and rates of polyploid formation in flowering plants. Annu Rev Ecol Syst 29: 467-501.

Rieseberg LH, Willis JH (2007). Plant speciation. Science 317: 910-914.

Scott R, Dagless E, Hodge R, Paul W, Soufleri I, Draper J (1991). Patterns of gene expression in developing anthers of Brassica napus. Plant Mol Biol 17: 195-207.

Soltis DE, Buggs RJA, Doyle JJ, Soltis PS (2010). What we still don't know about polyploidy. Taxon 59: 1387-1403.

Warwick SI, Simard M-J, Légère A, Beckie HJ, Braun L, Zhu B et al. (2003). Hybridization between transgenic Brassica napus L. and its wild relatives: Brassica rapa L., Raphanus raphanistrum L., Sinapis arvensis L., and Erucastrum gallicum (Willd.) O.E. Schulz. Theor App/ Genet 107: 528-539.

Supplementary Information accompanies this paper on Heredity website (http://www.nature.com/hdy) 